九州大学学術情報リポジトリ

Kyushu University Institutional Repository

Die Beziehung zwischen der geologischen Struktur und der Inkohlung im ChikuhoKohlenbecken, Japan

Takahashi, Ryohei

Faculty of Sciences, Kyushu University

https://doi.org/10.5109/1524318

出版情報：九州大學理學部紀要：Series D, Geology. 8 (1)，pp.1-18，1958-03-31. Faculty of Science, Kyushu University バージョン :

権利関係 : 
Mem. Fac. Sci., Kyushu Univ., Ser. D, Geology, Vol. VIII, No. 1, pp. 1-18, text-figs. 1-6, tables 1, 2, pls. 1-4, March 1958.

\title{
Die Beziehung zwischen der geologischen Struktur und der Inkohlung im Chikuho-Kohlenbecken, Japan.*
}

\author{
Von \\ Ryohei TAKAHASHI
}

\section{Einleitung}

Die genaue Bestimmung des Inkohlungsgrades ist eines der dringendsten Probleme, das die Kohlenforscher zu behandeln haben. Eine Reihe von Untersuchungsmethoden sind in dieser Richtung entwickelt worden. Es erscheint jedoch dem Verfasser nicht genügend klar, welche geologischen Vorgänge und Voraussetzungen in dem einen oder anderen Falle die Kohle zu ihrer Reife geführt haben.

Während des Inkohlungsprozesses, wobei wir die biochemische Phase von der geochemischen Phase unterscheiden, wirken die verschiedensten Faktoren auf die Kohle ein. Diese können nur bei genauer Kenntnis der geologischen Verhältnisse erklärt werden. Es ist wohl bekannt, dass sich die japanische Kohle von der aus anderen Ländern, z.B. der Ruhrkohle, petrographisch unterscheidet. Diese unterschiedliche fazielle Ausbildung ist noch keineswegs geklärt.

Verfasser hatte Gelegenheit, die Kyushu-Kohlen, Japan genauer, zu untersuchen. Dabei wurde es klar, dass eine enge Beziehung zwischen der geologischen Struktur des Kohlenbeckens und dem Inkohlungsverlauf besteht. Es liegt nahe, diese Erkenntnis von dem unten behandelten Chikuho-Kohlenbecken auf das gesamte Kyushu Kohlenbecken auszudehnen. Insofern sind die vorliegenden Untersuchungen lediglich als eine Vorarbeit zu werten.

Bei dieser Arbeit stand Herr Dr. H. Matsushita, Prof. der Kyushu Universität, mir mit Rat und Tat zur Seite. Ich spreche ihm hiermit meinen herzlichen Dank aus. Ihm verdanke ich auch die Kohlenproben aus einigen Bergwerken. Das chemische Labor von Fukuoka-Tsusansho in Wakamatsu hat dankenswerter Weise die Kohlenproben chemisch untersucht, was ich hier ebenfalls dankbar erwähnen möchte. Den grössten Teil dieser Arbeit habe ich im Geologischen Landesamt in Krefeld, Deutschland, bei Herrn Prof. Dr. E. STACH durchgeführt. Ich danke herzlich, dass mir die modernen Einrichtungen der Laboratorien zur Verfügung gestellt wurden, und dass Herr Prof. Dr. STACH mich stets in liebenswürdiger Weise unterstützt und beraten hat. Herr Prof. Dr. R. Potoní́, Frau Dr. M. TeichmüLleR

* Das Manuskript dieser Arbeit ist ein Teil eines Vortrages, der in Fukuoka anlässlich der Geologen Versammlung in West Japan, 1956, gehalten wurde.

* Received October 15, 1957. 


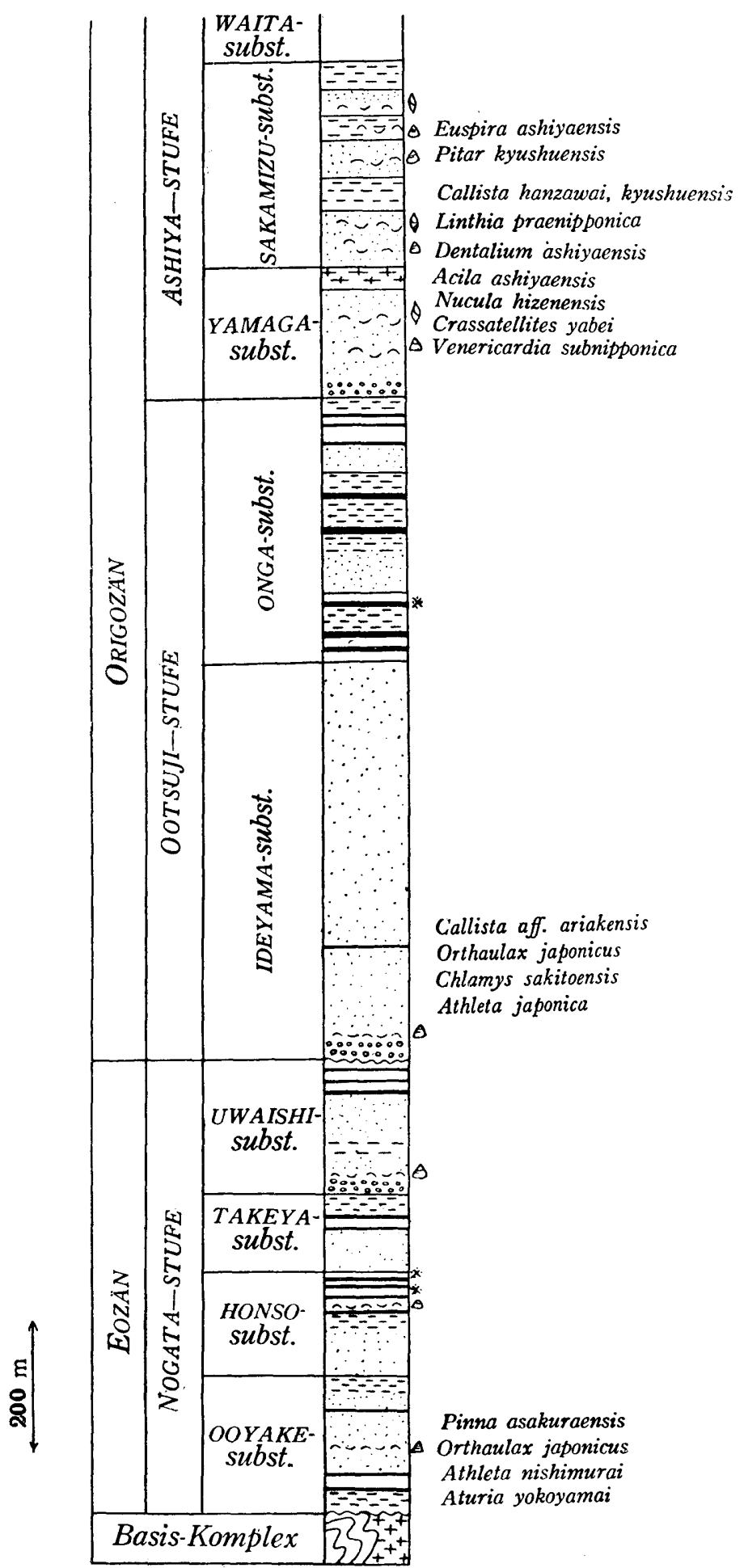

Abb. 1: Paläogen-Stratigraphie im Chikuho-Kohlenbecken. 
und Herr Dr. K. H. Josten im Geologischen Landesamt, Krefeld, haben mich freundlicherweise beraten, wofür ich Ihnen ebenfalls herzlich danke. Herr Dr. A. RABITz im Geologischen Landesamt, Krefeld, hat freundlicherweise mein Manuskript verbessert. Ihm spreche ich meinen herzlichen Dank aus.

\section{Geologische Übersicht.}

Bei Inkohlungsuntersuchungen ist es von Bedeutung, dass man die geologischen Verhältnisse des Kohlenbeckens genauer kennt. Sie tragen zum Verständnis der faziellen Ausbildung der Kohle bei. Die Geologie des Chikuho-Beckens ist früher von T. NAGAo und H. Matsushita eingehend behandelt worden. Danach wird das Paläogen hier in 3 Stufen eingeteilt: die produktive Noogata-, die produktive Otsujiund die sterile marine Ashiya-Stufe (Abb. 1).

Die Noogata-Stufe liegt diskordant auf dem Basiskomplex. Mit geringer Diskordanz liegt die Otsuji-Stufe über der Noogata. Als jüngste lagert die AshiyaStufe konkordant über der Otsuji. Die Noogata-Stufe ist nur im Südteil des Beckens vorhanden und zwar keilt sie allmählich nach Norden aus. Dagegen erreichen die beiden jüngeren Stufen im Nordteil des Beckens erhebliche Mächtigkeit. Bei Ebitsu lagert demzufolge die Otsuji-Stufe unmittelbar dem Basiskomplex auf. Die AshiyaStufe füllt im Süden des Beckens nur einen begrenzten Raum aus.

Wie aus der Abb. $1 \mathrm{zu}$ entnehmen ist, ist die Noogata-Stufe in 4, die OtsujiStufe in 2 und die Ashiya-Stufe in 3 Unterstufen gegliedert. Jede Unterstufe der Noogata-Stufe besteht aus einer Reihe von Ablagerungszyklen. Im ganzen sind die jüngeren Unterstufen reicher an Sandsteinen und Konglomeraten als die liegenden. Die Otsuji-Stufe besteht aus einem auffallenden "Mega-Zyklus" (namentlich von der Ideyama- zur Onga-Unterstufe). Da die Ashiya-Stufe marin ist, wird sie hier nicht eingehend behandelt. Zwischen der Noogata- und der Otsuji-Stufe hat eine orogene Bewegung stattgefunden. H. MATSUSHITA vermutet, dass die Noogata-Stufe in der "früheren Ariyake-See" und die Otsuji-Stufe in der "früheren Genkai-See" abgelagert wurde (Abb. 2).

Geologische Untersuchungen haben ergeben, dass das damalige Moor nicht immer ein geschlossenes Becken, sondern in verschiedene Teile gegliedert war. Zeitweise waren sie jedoch miteinander verbunden. Im allgemeinen streichen die Schichten NNW-SSE bis NW-SE. Dabei haben sie ein generelles Einfallen von $10-15^{\circ}$ nach NEE bzw. NE. Die ganze Struktur ist monoklin; es treten keine Falten auf. Im Westen überdecken diese gesamten tertiären Schichten diskordant den Basiskomplex, während sich an der Ostgrenze diese beiden Formationen an einem Bruch berühren, der NNW-SSE bis NW-SE streicht. Dieser Bruch wurde zeitlich im Tertiär angelegt und, wie später eingehend beschrieben wird, hat er besondere Bedeutung für die Inkohlung. Diese Störung kennzeichnet die geologische Karte dieses Gebietes, und H. Matsushita bezeichnet diese geologische Struktur als "Chikuho-Typ". Ausser 


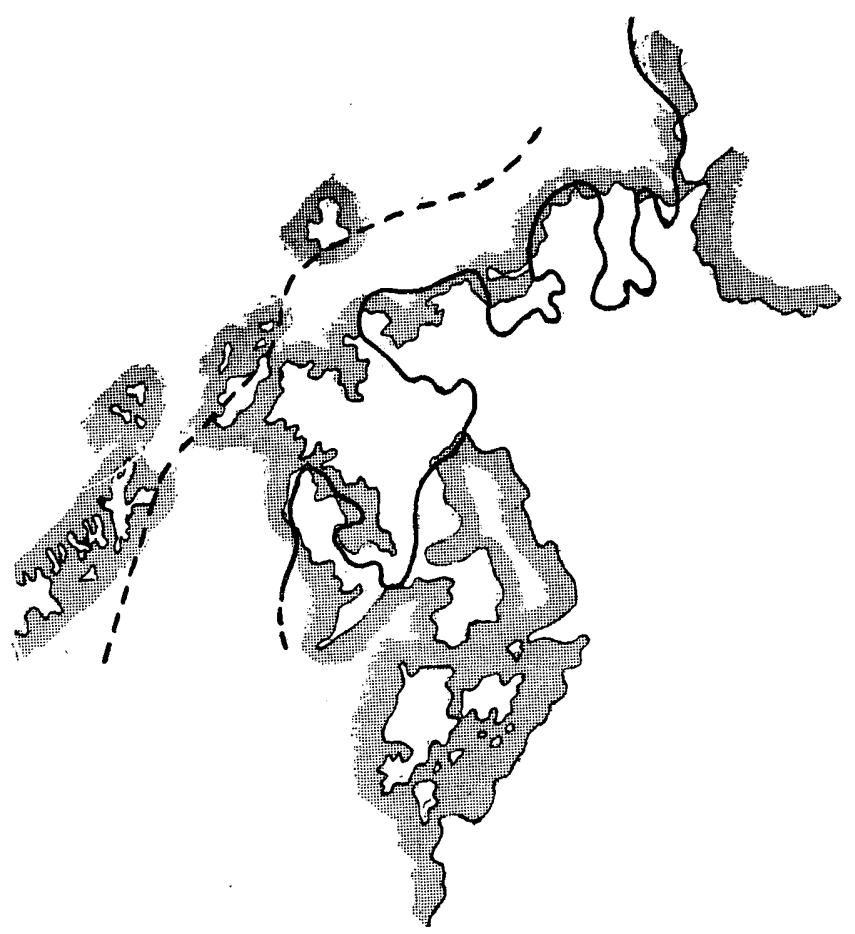

Paläogeographie der Ootsuji-Stufe

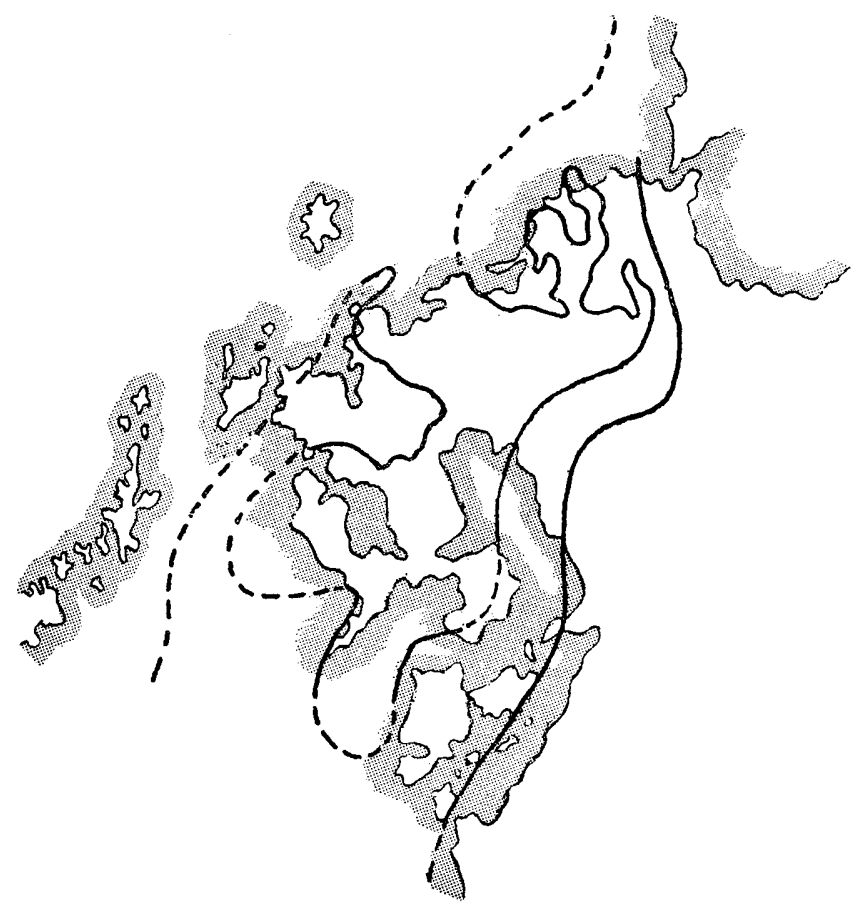

Paläogeographie der Ooyake-Stufe

Abb. 2: Paläogeographie der Noogata- und Ootsuji-Stufe 
durch diesen Bruch werden die Schichten noch durch einige kleinere Brüche in verschiedene Teile gegliedert. Echte Falten treten nicht auf.

\section{Das regionale Verhalten der Kohlen.}

Die Kohlenproben wurden in frischem Zustand aus dem Yoheda-Goshaku-Kohlenlager, aus der Schachtanlage Takamatsu im Nordgebiet, aus dem Yamahari-Kohlenlager der Schachtanlage Onoura im Mittelgebiet und aus dem Tagawa-YonshakuKohlenlager der Schachtanlage Tagawa im Südgebiet entnommen. Damit konnte gleichzeitig des Verhalten eines Flözes in horizontaler Erstreckung untersucht werden.

Die Schachtanlage Takamatsu liegt im nördlichsten Teil des Chikuho-Kohlenbeckens und hat einige Kohlenlager der Otsuji-Stufe abgebaut. Die Zeche Onoura, die in der Mitte des Beckens liegt, hat hauptsächlich die Kohlenflöze der HonsoUnterstufe aufgeschlossen. Die Tagawa-Schachtanlage im südlichsten Teil des Beckens baute bis jetzt auf den Kohlenflözen der Honso- bis Uwaishi-Unterstufe; jedoch nicht die tiefer lagernden Flöze der OyakeUnterstufe.

\section{a) Das Yoheda-Goshaku- Kohlenflöz.}

Die produktive Onga-Unterstufe wird in 3 Gruppen eingeteilt. Die unterste davon hat eine Mächtigkeit von etwa $100 \mathrm{~m}$. Das bedeutendste Kohlenflöz in diesen Sedimenten ist das Yoheda-Goshaku-Kohlenflöz. Es wird vom Schacht I nach Süden bis zum Schacht II etwa $9 \mathrm{~km}$. in der Streichrichtung abgebaut. Das Profil des Yoheda-Goshaku-Kohlenflözes ist in der Abbildung 3 wiedergegeben.

Wie schon erwähnt, werden die Kohlenflöze von einigen Brüchen, die

YOHEDA-GOSHAKI
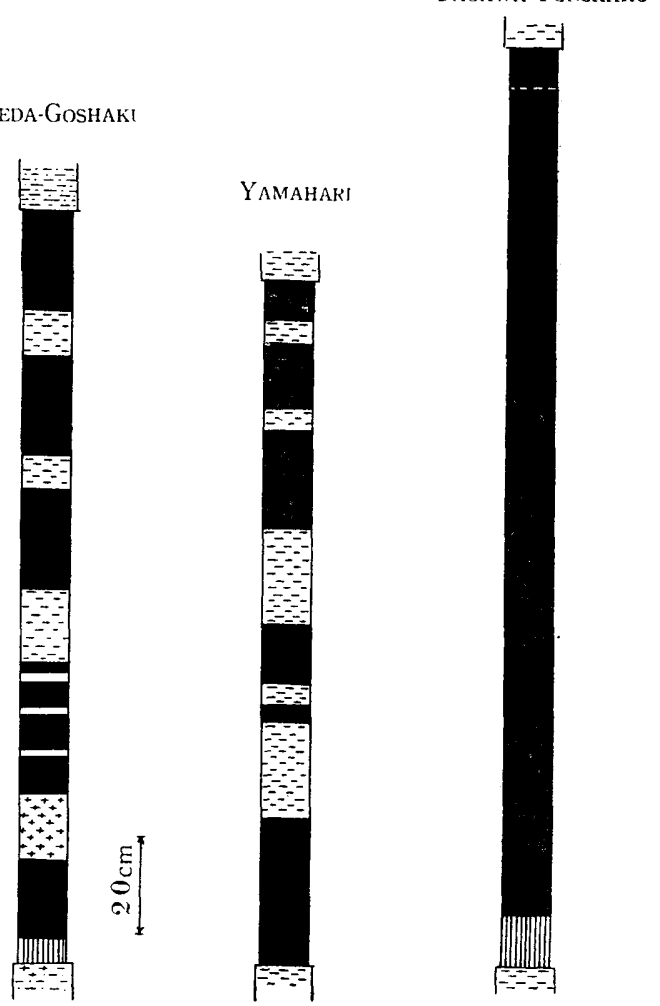

TAGaWa:YONSHaku

Abb. 3: Profil des Kohlenflözes. NNE-SSW streichen, versetzt. Die Proben wurden von tektonisch ungestörten Stellen entnommen. Im Hangenden des Yoheda-Goshaku-Kohlenflözes liegt meist ein 1.5-3.0 m. mächtiger Schieferton; stellenweise liegt unmittelbar über dem Flöz ein mittelkörniger Sandstein. In diesem Falle wird das Flöz meistens mächtiger 
und erreicht 1.7-1.9 m. In einigen Aufschlüssen wurde über der Kohle ein Konglomerat beobachtet. In diesem Falle zeigte das Flöz Auswaschungserscheinungen; Einzelheiten darüber sind dem Verfasser nicht bekannt und bedürfen einer näheren Untersuchung. Diese Schwankungen in der Mächtigkeit lassen jedoch keine direkte Beziehung zur geologischen Struktur des Kohlenbeckens erkennen.

Das Yoheda-Goshaku-Kohlenflöz ist streifenartig aufgebaut. Im oberen und im unteren Teil ist es besonders reich an Vitrit-Lagen, in denen linsengrosse Bernsteinkörner eingelagert sind. Diese Ausbildung hält in horizontaler Erstreckung aus und dient als Leitmerkmal.

\section{b) Das Yamahari-Kohlenflöz}

Das Yamahari-Kohlenflöz ist das oberste und ein bergbaulich wichtiges Flöz der Honso-Unterstufe. Die in der Oyake-Unterstufe enthaltenen Flöze sind besonders gut im Südgebiet entwickelt. Sie verlieren im mittleren Teil des Kohlenbeckens mehr und mehr an Bedeutung; d.h. die Mächtigkeit nimmt ab und mehr Bergepacken schieben sich ein. Dasselbe gilt für die Flöze, die in der Uwaishi-Unterstufe enthalten sind. Die Honso-Unterstufe ist deshalb in diesem mittleren Bezirk bergbaulich die wichtigste. Hinzu kommt, dass in dieser Honso-Unterstufe abbauwürdige Flöze liegen, die nach Norden hin auskeilen. Die Kohlenbildung wurde also am Südrand des Beckens durch Ton- und Sandschüttungen abgelöst. So kommt es, dass in diesem Gebiet 9 bauwürdige Flöze in nur $12 \mathrm{~m}$. mächtigen Ablagerungen liegen, während nach Süden diese Sedimente eine Mächtigkeit von 40-50 m. erreichen, wobei die Kohlenflöze an Bauwürdigkeit einbüssen.

Das Yamahari-Kohlenflöz wird von Grube 5 nach Grube 7 etwa $7.5 \mathrm{~km}$. in der Streichrichtung und $7.5 \mathrm{~km}$. senkrecht dazu abgebaut. Die durchschnittliche Mächtigkeit beträgt etwa $1.6 \mathrm{~m}$. Stellenweise steigt sie auf über $4 \mathrm{~m}$. an. Die Ursachen dieser starken Schwankungen sind noch nicht genau untersucht; man kann annehmen, dass in dem Zentrum des Gebietes die Kohle noch mächtiger entwickelt sein wird. Diese ist aber noch nicht aufgeschlossen und wird erst in einer grösseren Tiefe erreicht.

Das Flöz selbst ist streifenartig ausgebildet und besonders reich an Mattkohle, daher ist die Gefügefestigkeit grösser als bei den oben erwähnten Flözen. Über der Kohle liegt meistens ein grauer Sandschiefer oder feinkörniger Sandstein.

\section{c) Das Tagawa-Yonshaku-Kohlenflöz}

Die Zeche Tagawa hat 4 Schachtanlagen. Von jeder dieser Anlagen wird ein anderes Flöz abgebaut. Es ist daher nicht möglich, von einem Kohlenflöz Proben zu entnehmen, die in grösserer Entfernung voneinander liegen. Die Untersuchungen an diesem Flöz stützen sich daher nur auf wenige Proben, im Gegensatz zu den anderen oben beschriebenen Kohlenflözen. Wie schon erwähnt, sind in allen Schacht- 
anlagen der Zeche Tagawa keine Flöze der Oyake-Unterstufe sondern die Kohlen der Honso- bis Uwaishi-Unterstufe aufgeschlossen. Das Tagawa-Yonshaku Kohlenflöz ist das 2. unten von in der Honso-Unterstufe und wird in der Schachtanlage 3 abgebaut. Ausser diesem sind noch folgende Flöze von wirtschaftlich grösserer Bedeutung: Tagawa-Sanjaku-, Tagawahasshaku-, Shinsanjaku-Kohlenflöz.

Sie sind gut im Südgebiet entwickelt; aber im Vergleich zu dem mittleren, dem Onoura-Gebiet sind die Abstände zu den einzelnen Flözen grösser. Ausserdem ist die Kohle unreiner ausgebildet durch Bergmittel. Die Flöze im Tagawa-Gebiet werden ebenso wie die in den anderen Gebieten durch die NNW-SSE bis NW-SE streichenden Störungen versetzt.

Alle Flöze der Tagawa-Zeche sind im Süden stärker als im Norden ausgebildet. Der Aufbau des Tagawa-Yonshaku-Flözes ist in der Abb. 3 wiedergegeben.

\section{Die Bestimmung des Inkohlungsgrades durch die Kurzanalyse.}

Wie schon früher erwähnt, wurden von jedem Flöz verschiedene Proben in unterschiedlicher Teufe entnommen. Dabei wurde beachtet, dass die Schlitzproben aus dem Gesamtprofil der Flöze auch wirklich dem Durchschnitt entsprechen. In der Tafel 1 sind die Kurzanalysen von Vitrit wiedergegeben.

Wie die Tafel zeigt, gehören die meisten Proben in den Bereich der Gasflamm-/ Gaskohle; einige sind sogar noch als Braunkohlen anzusprechen, z.B. die Proben T-7, T-8 und T-11 aus der Takamatsu-Zeche. Dasselbe gilt für die Proben A-2, B-2, C-3 und D-4 aus der Onoura-Zeche. Diese Proben haben 41-43\% flüchtige Bestandteile und gehören nach dem Gehalt an flüchtigen Bestandteilen zu den Braunkohlen. Im mikroskopischen Bild lassen diese Proben jedoch erkennen, dass sie sich nicht von anderen unterscheiden. Die Farbe des Resinits und des Sporinits ist bei allen Proben die gleiche. Die Anreicherung des Resinits im Vitrit scheint den Analysenwert zu beeinflussen, weshalb die Inkohlungsgrade dieser viele flüchtige Bestandteile enthaltenden Proben nicht besonders niedrig sind.

In der Abb. 4 sind die chemischen Daten in Beziehung gesetzt zu der Teufe. Die Kurve der flüchtigen Bestandteile des Yoheda-Goshaku-Kohlenflözes verläuft gänzlich anders als die der anderen Flöze. Wie M. TEICHмüLLER berichtet, gibt es keinen gültigen Massstab, der in allen Inkohlungsstadien gleichartig brauchbar ist. Die Braunkohlen, Flamm- und Gasflammkohlen werden am besten durch den Wassergehalt der lufttrockenen Kohle klassifiziert. Andererseits wird für höher metamorphe Kohlen (Fett-, Magerkohlen und Anthrazit) der Gehalt an flüchtigen Bestandteilen der beste Inkohlungsmassstab. In diesem Inkohlungsbereich spalten sich polare Gruppen von Aromatkernen als flüchtige Bestandteile ab. Die Spaltung der nichtaromatischen Gruppen hat für sich selbst eine Bedeutung für die Inkohlung. Damit hat die Bestimmung der flüchtigen Bestandteile meines Erachtens nach als Inkohlungsmassstab besondere Bedeutung. 
Zahlentafel 1: Kurzanalyse von Vitrit.

\begin{tabular}{|c|c|c|c|c|c|c|c|c|}
\hline Zeche & Flözname & Probe Nr. & Tief (m) & $\begin{array}{r}\text { Wasser } \\
\%\end{array}$ & $\begin{array}{r}\text { fl. Best. (af) } \\
\% \text { ) }\end{array}$ & $\underset{\%}{\text { Asch }}$ & Heizwert & $\mathrm{S}_{\%}$ \\
\hline Takamatsu & 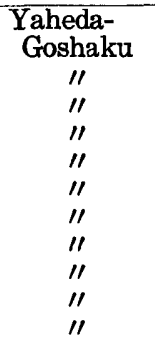 & $\begin{array}{l}\mathrm{T}-1 \\
\mathrm{~T}-2 \\
\mathrm{~T}-3 \\
\mathrm{~T}-4 \\
\mathrm{~T}-5 \\
\mathrm{~T}-6 \\
\mathrm{~T}-7 \\
\mathrm{~T}-8 \\
\mathrm{~T}-9 \\
\mathrm{~T}-10 \\
\mathrm{~T}-11\end{array}$ & $\begin{array}{l}443 \\
500 \\
233 \\
254 \\
246 \\
240 \\
700 \\
700 \\
300 \\
501 \\
790\end{array}$ & $\begin{array}{l}6.27 \\
7.04 \\
8.35 \\
8.54 \\
7.14 \\
6.73 \\
5.15 \\
5.35 \\
7.74 \\
7.22 \\
6.08\end{array}$ & $\begin{array}{l}38.45 \\
37.48 \\
34.60 \\
35.77 \\
36.28 \\
37.26 \\
44.41 \\
41.89 \\
35.65 \\
37.18 \\
45.23\end{array}$ & $\begin{array}{l}2.38 \\
2.43 \\
1.03 \\
1.16 \\
2.32 \\
1.85 \\
3.47 \\
2.24 \\
2.28 \\
3.31 \\
2.79\end{array}$ & $\begin{array}{l}7063 \\
6927 \\
6890 \\
6806 \\
6956 \\
7081 \\
7186 \\
7298 \\
6729 \\
6755 \\
7199\end{array}$ & $\begin{array}{l}0.506 \\
0.556 \\
0.507 \\
0.436 \\
0.333 \\
0.390 \\
0.467 \\
0.714 \\
0.390 \\
0.489 \\
0.724\end{array}$ \\
\hline Onoura & 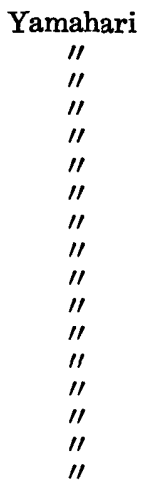 & $\begin{array}{l}A-2 \\
A-3 \\
A-4 \\
A-5 \\
A-6 \\
B-1 \\
B-2 \\
B-4 \\
C-1 \\
C-2 \\
C-3 \\
D-1 \\
D-2 \\
D-3 \\
D-4 \\
D-5 \\
D-6\end{array}$ & $\begin{array}{l}384 \\
318 \\
458 \\
383 \\
384 \\
151 \\
201 \\
196 \\
111 \\
253 \\
284 \\
197 \\
311 \\
336 \\
255 \\
358 \\
196\end{array}$ & $\begin{array}{l}2.50 \\
3.75 \\
3.76 \\
3.67 \\
3.67 \\
3.94 \\
2.80 \\
3.79 \\
3.37 \\
3.65 \\
1.97 \\
4.05 \\
3.86 \\
3.88 \\
3.97 \\
4.86 \\
4.95\end{array}$ & $\begin{array}{l}43.24 \\
37.40 \\
34.97 \\
35.60 \\
34.33 \\
35.85 \\
41.20 \\
36.62 \\
33.08 \\
36.23 \\
42.26 \\
35.32 \\
35.12 \\
36.58 \\
36.84 \\
34.38 \\
35.62\end{array}$ & $\begin{array}{r}11.57 \\
4.14 \\
5.31 \\
2.32 \\
3.00 \\
1.81 \\
4.82 \\
3.59 \\
4.61 \\
5.90 \\
8.55 \\
2.97 \\
3.05 \\
4.14 \\
4.62 \\
5.73 \\
5.90\end{array}$ & $\begin{array}{l}7060 \\
7420 \\
7140 \\
7600 \\
6003 \\
7720 \\
7570 \\
7520 \\
7500 \\
7410 \\
7540 \\
7530 \\
7530 \\
7500 \\
7330 \\
7520 \\
7060\end{array}$ & \\
\hline \multirow{4}{*}{ Tagawa } & \multirow{4}{*}{$\begin{array}{c}\text { Ita- } \\
\text { Goshaku } \\
\text { " } \\
\text { " } \\
\text { Tagawa- } \\
\text { Yonshaku } \\
\text { " } \\
\text { " } \\
\prime \prime \\
\text { " } \\
\prime \prime\end{array}$} & M-1 & 206 & 4.23 & 41.23 & 3.36 & 7320 & \\
\hline & & $\begin{array}{l}\mathrm{M}-2 \\
\mathrm{M}-3\end{array}$ & $\begin{array}{l}218 \\
218\end{array}$ & $\begin{array}{l}3.68 \\
3.98\end{array}$ & $\begin{array}{l}44.10 \\
42.02\end{array}$ & $\begin{array}{l}6.42 \\
2.70\end{array}$ & $\begin{array}{l}7330 \\
7670\end{array}$ & \\
\hline & & M-4 & $\begin{array}{l}504 \\
538\end{array}$ & $\begin{array}{l}4.43 \\
4.33\end{array}$ & $\begin{array}{l}35.43 \\
36.42\end{array}$ & $\begin{array}{l}2.23 \\
2.25\end{array}$ & $\begin{array}{l}7546 \\
7520\end{array}$ & \\
\hline & & $\begin{array}{l}M-6 \\
M-7 \\
M-8 \\
M-9\end{array}$ & $\begin{array}{l}503 \\
494 \\
423 \\
575\end{array}$ & $\begin{array}{l}4.47 \\
4.19 \\
4.65 \\
3.93\end{array}$ & $\begin{array}{l}34.22 \\
39.33 \\
36.53 \\
39.52\end{array}$ & $\begin{array}{l}2.13 \\
3.06 \\
2.39 \\
2.26\end{array}$ & $\begin{array}{l}7400 \\
7490 \\
7490 \\
7630\end{array}$ & \\
\hline
\end{tabular}

Drückt man den Inkohlungsgrad mit Hilfe der flüchtigen Bestandteile aus, dann nimmt die Metamorphose der Kohle der Zeche Takamatsu nach der Tiefe allmählich ab. Diese Tatsache steht den bisher bekannten Ergebnissen gegenüber; M. TEICHMüLlER hat Abweichungen von der HILT'schen Regel beschrieben. Die Kurve des Tagawa-Yonshaku- und des Yamahari- Kohlenflözes zeigen dagegen einen normalen Verlauf. Da das Yoheda-Goshaku Kohlenflöz junger als die beiden anderen Flöze ist, ware anzunehmen, dass seine Inkohlungskurve durch weiter fortschreitende Inkohlung einen Verlauf erreichen konnte, der demjenigen der anderen Flöze ähnlich ist.

Der Inkohlungssprung, den E. STACH und K. LEHMANN als erste beschrieben haben, ist von einigen Forschern chemisch bestätigt worden. Nach E. STACH und M. Th. Mackowsky liegt der Inkohlungssprung zwischen 29 und 30\% flüchtigen Bestandteilen. K. PatTeisky berichtet, dass der Inkohlungssprung der Ruhrkohlen bei 29.5\% flüchtigen Bestandteilen liegt. Es ist auch noch problematisch, ob dieser 


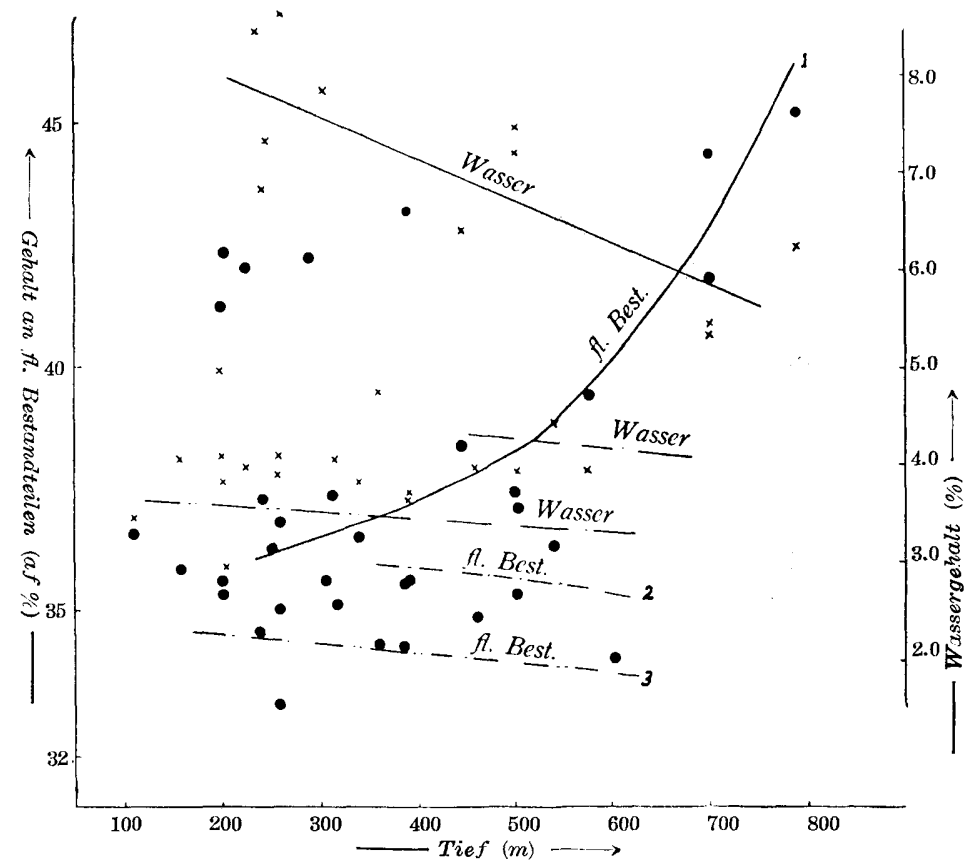

Abb. 4: Verhältnis zwischen der Tiefe und dem Gehalt an Wasser, fi. Bestandteilen.

Inkohlungssprung tatsächlich in allen Kohlenbecken ausserhalb des Ruhrgebietes vorhanden ist. Nach dem Ergebnis, das K. AsAI und K. TANNo berichtet haben, gibt es auch in japanischen Kohlen ein "alteration point of coalification", der bei einem Kohlenstoffgehalt von $83 \%$ liegt. K. NAKAMURA berichtet, dass dieser "alteration point of coalification" bei $86 \%$ Kohlenstoffgehalt liegt. Unter Berücksichtigung von unterschiedlichen Inkohlungsbedingungen kann man den Inkohlungssprung als identisch mit dem "alteration point of coalification" ansehen. Der Inkohlungssprung ist in japanischen Kohlen bei etwa $40 \%$ flüchtigen Bestandteilen nachgewiesen worden. Verfasser hat den Gehalt an flüchtigen Bestandteilen des Vitrits mit dem des Durits nicht verglichen; deshalb können an dieser Stelle keine besonderen Angaben darüber gemacht werden.

Man muss hier nicht nur den Gehalt an flüchtigen Bestandteilen sondern auch den Wassergehalt berücksichtigen. Im allgemeinen sinkt der natürliche Wassergehalt lufttrockener Steinkohlen vom Flamm- bis Fettkohlenstadium von ca. 5\% auf $1 \%$ herab und nimmt dann beim Anthrazit wieder etwas zu. Da der Wassergehalt von der Grösse der inneren Oberfläche abhängig ist, nimmt dieser im Laufe des Inkohlungsprozesses ab, um letztlich im Anthrazitstadium wieder zuzunehmen. Im allgemeinen nimmt der Wassergehalt um $1 \%$ auf $30 \mathrm{~m}$. Teufe ab. Der Wassergehalt der Braunkohlen von Borneo und der Pechkohlen vom Alpenvorland nimmt bei $100 \mathrm{~m}$. Teufenzunahme um 0.5-1.0\% ab. Ebenso verhalten sich die YamahariKohlen und die anderen beiden Flöze, bei denen die Abnahme ca. 0.5\% auf $100 \mathrm{~m}$. 
Teufe beträgt. Der Wassergehalt wird auffallend stark beeinflusst durch Druck, Temperatur und Zeit. Nach dem Verlauf der Inkohlung des Yoheda-GoshakuKohlenflözes ist die Metamorphose in grosser Teufe weniger stark, obgleich sie natürgemäss dort höheren Druck- und Temperatur- Bedingungen ausgesetzt war. Auf die Ursachen, die dieses aussergewöhnliche Verhalten der Yoheda-GoshakuKohle bedingen, wird später noch näher eingegangen werden.

\section{Mikroskopische Untersuchungen}

Alle diese Kohlenproben wurden unter dem Auflichtmikroskop untersucht Ein grosser Vorteil, die Probe im Auflicht zu untersuchen, liegt darin, dass man gleichzeitig durch Reflexionsmessung den Inkohlungsgrad bestimmen kann. Die Kohlenstücke werden in Kunstwachs (Polyäster) eingebettet und poliert.

\section{a: Beschreibung}

Im folgenden sollen die mikroskopischen Beobachtungen, die an den einzelnen Flözen gemacht wurden, kurz beschrieben werden. Der Vitrit zeigt eine ausgesprochen gute Zellstruktur. Das tritt besonders deutlich hervor, wenn die Zellräume resinitisch durchtränkt sind. Im Vitrinit befindet sich der Resinit oftmals in linigem Arrangement, dieser spaltet sich einmal aus dem Zellraum und arrangiert sich wieder. Diese Anordnung des Resinits scheint mehr oder weniger Beziehung zu der Teufe zu haben. Im Zellhohlraum des Vitrits gibt es auch stellenweise eine Menge Verunreinigungen. Diese dürften sich technologisch als Vitrinertit verhalten. Insofern hat die tonige Substanz besondere Bedeutung.

An dem Durit der einzelnen Kohlenflöze lässt sich kein Unterschied erkennen. Durit besteht aus Degradinit als Grundmasse und aus Exinit, Vitrinit und Resinit als Nebenbestandteilen. Der in dem Durit enthaltene Vitrinit ist in den meisten Fällen gut kolloidiert und zum Collinit geworden, obgleich er noch mehr oder weniger häufig Telinitstücke enthält. Zwei verschiedene Resinitarten sind zu erkennen; dabei ist die eine Resinitart immer stärker entfärbt als die andere.

Die Farbe des Resinits im Durit ist immer dunkler als die im Vitrit, und das zeigt, dass der Resinit im Durit im Vergleich zu dem im Virit weniger hoch inkohlt ist. Im Exinit sind Mikrosporen am bedeutendsten. Sie sind 1-5 $\mu$ gross $(2-3 \mu$ am häufigsten). Dabei kann man verschiedene Arten erkennen, die aber nicht einwandfrei zu bestimmen sind. Daher werden nur die Tenui- von den Crassi-sporen unterschieden. Die Verteilung dieser beiden Arten im Flözprofil ist unregelmässig, daher stratigraphisch nicht mittelbar zu verwenden. Die Kutikulen sind als akzessorische Bestandteile vorhanden. Gelegentlich treten die Kutikulen gehäufter auf und bilden dann einen typischen Kutikulen-Clarit. Im Sklerotinit sind Pilzfruchtkörper von Bedeutung. Am Reflexionsvermögen erkennt man zwei Arten von Pilzfruchtkörpern: die eine hat niedrigere Reflexion als der Vitrit, die andere dagegen reflek- 
tiert etwas höher. Zur Zeit ist es dem Verfasser noch unklar, ob diese zwei Arten auf biologische Unterschiede zurückzuführen oder lediglich durch die Inkohlung bedingt sind. Ausser diesen Pilzkörpern sind mehr oder weniger Teleutosporen vorhanden.

Im Durit und Vitrit befinden sich auch Fadenpilze oder Bakterien, die weisse, feine, stückartige Splitterform haben.*

Im allgemeinen hat der Durit eine grosse Menge Verunreinigungen. Reiner Durit ist sehr selten. Die häufigste Verunreinigung ist Tonsubstanz, dann folgen PyritKonkretionen. Quarzkörner, Kalkspat und Eisenspat treten selten auf.

\section{a-1: Yoheda-Goshaku Kohlenflöz}

Vitrit gehört meistens zu Telinit. Innerhalb der Duritbänder jedoch befinden sich einige Vitritbändchen, die zu Collinit umgewandelt sind. Vitrinit im Durit ist auch in den meisten Fällen zu Collinit umgeformt. Telinit enthält oftmals viele Resinitkugeln, so dass er als technischer Clarit benannt werden muss. Resinit im Vitrit ist "in situ" in Zellhohlräumen enthalten, zum Teil isoliert und zum Teil rearrangiert in Linien. In einigen Teliniten befinden sich mehrere sehr feine, weisse, stückartige Bakterien oder Fadenpilze, die ein höheres Relief als das des Vitrits zeigen. Der Durit jeder Kohlenprobe besteht aus den gleichen Bestandteilen, und es besteht kein auffallender Unterschied in Duritproben aus verschiedener Teufe. Dies ist darauf zurückzuführen, dass sich die pflanzlichen Stoffe in Unabhängigkeit von der gegenwärtigen Tiefe abgelagert haben. Das gilt auch für Clarit und Vitrit. Nur Schwefelkies bildet eine Ausnahme, d.h., er wird weiter vorherrschender nach der Tiefe der Entnahmestelle; und das stimmt mit dem Ergebnis der Kurzanalyse überein. Andererseits sind die Mikrostreifen nach der Tiefe allmählich stärker gestört worden; die Grenzlinie zwischen den Streifenarten ist nicht linig sondern ausgesprochen gebogen und wellenförmig.

Als Ursache dieser Lagerung sind Gleitungen anzusehen, die man oft bei anderen Sedimentgesteinen erkannt hat. In grösserer Tiefe ist diese Erscheinung öfters zu beobachten. Resinit und Mikrosporen sind Hauptmazeral im Exinit; die Farbe von Resinit ist schwächer als die von Mikrosporen; die Farbe der Mikrosporen ist in jeder Probe fast gleichartig. So ist kein Unterschied zwischen den Farben der Proben zu ersehen. In Probe Nr. 2 und 5 handelt es sich um eine Menge von Kutikulen, und auch in Probe Nr. 3, 4, 7, 8 und 10 befinden sich mehr oder weniger Kutikulen. Es gibt, wie erwähnt, keine Beziehung zwischen der Tiefe und der vorkommenden Menge der Kutikulen.

\section{a-2: Yamahari Kohlenflöz}

Die Proben sind aus seichter Stelle weniger als $400 \mathrm{~m}$. Tiefe entnommen worden,

* Auf die Einzelheiten dieses bakterienartigen Mazerals soll in einem anderen Bericht näher eingegangen werden. 
und so kommt hier die klare Beziehung zwischen der Tiefe und der Mikrobilder, wie in Yoheda-Goshaku Kohlenflöz, nicht zum Ausdruck. Trotzdem besteht ein Unterschied zwischen den Proben von mehr als $250 \mathrm{~m}$. Tiefe und höher gelegenen; namentlich bei Probe C-1, B-1, 2, D-1, 6 sind die Mikrostreifen im allgemeinen regelmässig und ausserdem tritt noch feinkörniger Mikrinit auf, den man trotz der geringen Menge gut erkennen kann. Auch bei Probe D-6 ist der Vitrit zum Teil zum Semifusinit geworden, infolgedessen mag es sein, dass das Ausgangsmaterial bei dem biochemischen Prozesse mehr oder weniger stark oxydiert wurde. Andererseits sind bei tiefer liegenden Proben, wie Probe D-3, 5, die Mikrostreifen ziemlich gestört und viele Pyrit-Konkretionen zur Ablagerung gelangt. Einige Proben zeigen oftmals von dieser allgemeinen Neigung abweichende mikroskopische Bilder, aber in ganzen gesehen, kann man die Proben von tieferen Lagen von den höher als $250 \mathrm{~m}$. liegenden unterscheiden.

\section{a-3: Tagawa-Yonshaku-Kohlenflöz}

$\mathrm{Da}$ die Proben zwischen 500-600 m. Tiefe entnommen wurden, kommt eine klare Beziehung zwischen der Tiefe und der Mikrobilder, wie im Falle des YohedaGoshaku-Kohlenflözes, nicht zum Ausdruck. Im allgemeinen ist Harz in den Proben, die die schönen Streifenbilder zeigen, linienförmig angeordnet. Es handelt sich bei diesen Proben um solche mit geringen Mengen von Mikrinitkörnchen.

So mag die Beziehung zwischen der Tiefe und den Mikrobildern, die von der oben beschriebenen beiden Kohlenproben vermutet worden ist, auch für das TagawaYonshaku-Kohlenflöz gültig sein.

Vitrit gehört meistens zum Telinit. Im Durit ist viel Exinit und Sklerotinit eingelagert. Im Exinit sind die Mikrosporen bedeutend, und die Kutikulen befinden sich nur in einigen Horizonten. Die tonige Substanz ist hauptsächlich Verunreinigung. Pyrit ist sehr selten, nur in einigen Proben treten kleinere Stückchen auf.

\section{b: Reflexionsmessung}

Nach dem Gehalt an flüchtigen Bestandteilen gehören die betreffenden Kohlen hauptsächlich zur Gasflammkohle und zum Teil zur Gas- und Flammkohle. Im übrigen schwankt bei diesen niedrig inkohlten Kohlen die Beziehung zwischen dem Reflexionsvermögen und dem Gehalt an flüchtigen Bestandteilen in mehr oder weniger breitem Bereich. Wie von der Zahlentafel-2 zu ersehen, ist das Verhältnis des Reflexionsvermögens zur Tiefe nicht klar. Es ist jedoch leicht zu ersehen, dass das Verhältnis zwischen dem Gehalt an flüchtigen Bestandteilen und dem Reflexionsvermögen von den zur Zeit bekannten Werten für Gas- und Gasflammkohlen abweicht. Die Kohlen von weniger als $42 \%$ fl. Bestandteilen haben ein niedrigeres Reflexionsvermögen als die Kohlen, welche SEYLER beschreibt. Das ist auch eine Eigentümlichkeit der japanischen Kohle.

Im Diagramm R/H : C zeigt die japanische Kohle im allgemeinen die niedrigste 
Zahlentafel 2: Reflexionsmessung.

\begin{tabular}{|c|c|c|c|c|c|}
\hline Zeche & Flözname & Probe Nr. & Tief (m) & fl. Best. (\%) & Reflex. \\
\hline Takamatsu & $\begin{array}{l}\text { Yoheda-Goshaku } \\
\text { Kohlenflöz }\end{array}$ & $\begin{array}{l}\mathrm{T}-1 \\
\mathrm{~T}-2 \\
\mathrm{~T}-3 \\
\mathrm{~T}-4 \\
\mathrm{~T}-5 \\
\mathrm{~T}-6 \\
\mathrm{~T}-7 \\
\mathrm{~T}-8 \\
\mathrm{~T}-9 \\
\mathrm{~T} 10 \\
\mathrm{~T} 11\end{array}$ & $\begin{array}{l}443 \\
500 \\
233 \\
254 \\
245 \\
240 \\
700 \\
700 \\
300 \\
501 \\
790\end{array}$ & $\begin{array}{l}38.45 \\
37.48 \\
34.60 \\
35.77 \\
36.28 \\
37.26 \\
44.41 \\
41.89 \\
35.65 \\
37.18 \\
45.23\end{array}$ & $\begin{array}{l}0.71 \\
0.71 \\
0.74 \\
0.78 \\
0.74 \\
0.71 \\
0.64 \\
0.71 \\
0.71 \\
0.71 \\
0.71\end{array}$ \\
\hline Ônoura & $\begin{array}{l}\text { Yamahari- } \\
\text { Kohlenflöz }\end{array}$ & $\begin{array}{l}\text { A-2 } \\
\text { A-3 } \\
\text { A-4 } \\
\text { A-5 } \\
\text { B-1 } \\
\text { B-2 } \\
\text { B-4 } \\
\text { C-1 } \\
\text { C-2 } \\
\text { C-3 } \\
\text { D-1 } \\
\text { D-2 } \\
\text { D-3 } \\
\text { D-4 } \\
\text { D-5 } \\
\text { D-6 }\end{array}$ & $\begin{array}{l}384 \\
318 \\
458 \\
383 \\
151 \\
201 \\
196 \\
111 \\
253 \\
284 \\
197 \\
311 \\
336 \\
255 \\
358 \\
196\end{array}$ & $\begin{array}{l}43.24 \\
37.40 \\
34.97 \\
35.60 \\
35.85 \\
41.20 \\
36.68 \\
33.08 \\
36.28 \\
42.26 \\
35.32 \\
35.12 \\
36.58 \\
36.84 \\
34.38 \\
35.62\end{array}$ & $\begin{array}{l}0.74 \\
0.71 \\
0.85 \\
0.71 \\
0.74 \\
0.67 \\
0.67 \\
0.78 \\
0.85 \\
0.71 \\
0.71 \\
0.78 \\
0.78 \\
0.74 \\
0.71 \\
0.71\end{array}$ \\
\hline Tagawa & $\begin{array}{c}\text { Tagawa-Sanjaku } \\
\text { Kohlenflöz }\end{array}$ & $\begin{array}{l}M-4 \\
M-5 \\
M-6 \\
M-7 \\
M-8 \\
M-9\end{array}$ & $\begin{array}{l}504 \\
538 \\
603 \\
494 \\
423 \\
575\end{array}$ & $\begin{array}{l}35.43 \\
36.42 \\
34.22 \\
39.38 \\
36.53 \\
39.52\end{array}$ & $\begin{array}{l}0.78 \\
0.78 \\
0.71 \\
0.78 \\
0.81 \\
0.67\end{array}$ \\
\hline
\end{tabular}

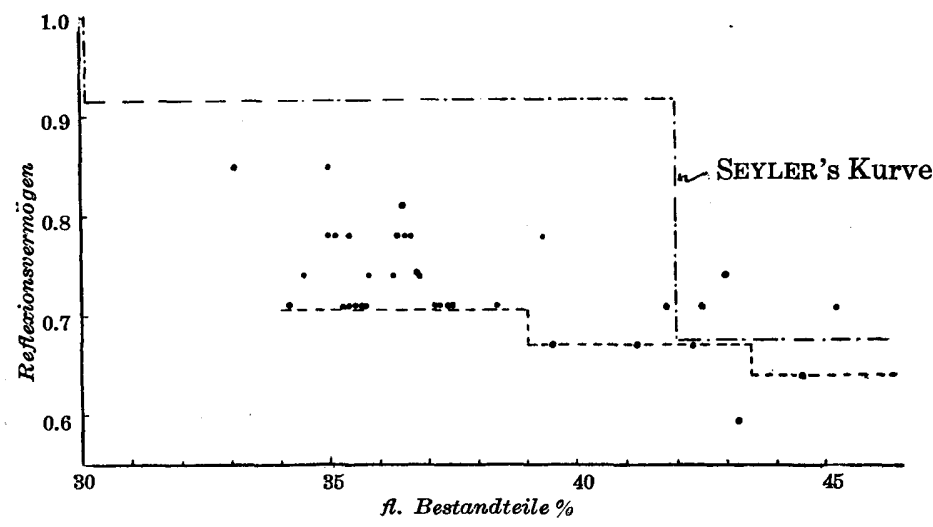

Abb. 5: Verhältnis zwischen dem Reflexionsvermögen und den Gehalt an fl. Bestandteilen.

Inkohlungskurve. Im Vergleich zu ausländischen Kohlen mit gleichem C-Gehalt enthalten die japanischen Kohlen relativ mehr H-Stoff.

Als Gründe dafür sind anzugeben:

1) Japanische Kohle ist mehr hydroaromatisch, 
2) Japanische Kohle hat viele Alkyl-Seitenketten oder diese Ketten sing relativ länger,

3) In der japanischen Kohle ist der Aromat-Kern vor allem klein.

Auf Grund der verschiedenen chemischen Untersuchungen, z.B. durch die röntgenographischen sowie magnet-chemischen Untersuchungen der Bitumina und Pseudobitumina der Yubari-Kohlen in Hokkaido, ist zu vermuten, dass der aromatische Kern aus einem von 3-4 Benzenringen zusammengesetzten Komplex besteht, und dieser Komplex mit der sehr komplizierte Sauerstoff enthaltenden Brücke verbunden ist. Das Reflexionsvermögen nimmt mit der Aromatisierung und Kondensation des Huminkomplexes zu. Nach der Reflexionsmessung scheint diese Vermutung auch richtig zu sein.

\section{Beziehung zwischen der geologischen Struktur und der Inkohlung.}

\section{a: Allgemeiner Verlauf der Inkohlung.}

Trotz einer Fülle von Untersuchungen durch Chemiker, Physiker und Geologen, ist das Inkohlungsproblem noch nicht vollkommend geklärt worden. Der eine hält die biologische Stufe für am bedeutendsten, der andere dagegen hält die nachfolgende geochemische Phase für am wichtigsten. M. Th. Mackowsky hat in ihrem Aufsatz über die Inkohlungsfrage beschrieben, dass im Verlauf der 1. Phase der Inkohlung CAnreicherungen zwischen 30-40\% erfolgen können und gleichzeitig während dieser Phase die Kohlenbestandteile entstehen, die sich um fast $15 \%$ Kohlenstoff voneinander unterscheiden. M. TEICHMÜLLER hält andererseits die geochemische Stufe für am wichtigsten und hat diese in den präorogenischen, synorogenischen und postorogenischen Ablauf gegliedert.* Nach dem Stand der heutigen Erkenntnis ist es noch

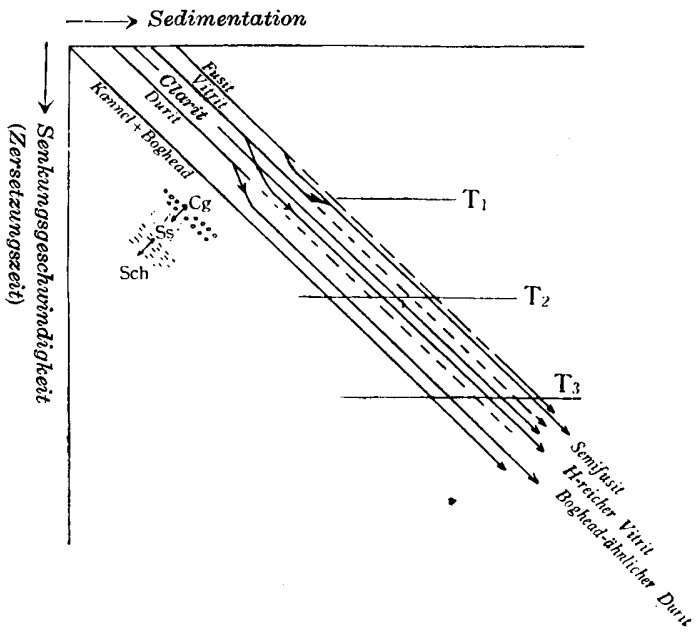

Abb. 6: Schematische Darstellung: Verhältnis zwischen der Geschwindigkeit der Ablagerung des Materials und der der Absenkung des Bodens. schwer zu entscheiden, welche Theorie die richtige ist. Trotzdem aber möchte der Verfasser hier betonen, dass man die erste Stufe nicht unterschätzen darf. Es gibt übrigen eine bestimmte Beziehung zwischen der Geschwindigkeit der Ablagerung des angeschwemmten pflanzlichen Materials und der der Absenkung des Bodens.

* In der präorogenischen Metamorphose hat M. TEICHüLler die erste Stufe und gewissermassen anfängliche Inkohlung der 2-Stufe (sogenannten diagenetische Inkohlung) umfasst. 
Sinkt der Boden zu schnell ab, so entspricht dann nicht mehr die Menge des transportierten pflanzlichen Materials der Bodenabsenkung, und infolgedessen kommen anstatt der pflanzlichen Stoffe Schiefertone und Sandsteine zur Ablagerung.

Im Gegenfall wird die Ablagerung des Torfes unterbrochen. In Anlehnung an bisherige Untersuchungen soll der Ausgangsstoff des Zersetzungsfusinits an relativ trockenen Stellen ablagert und bei relativ langzeitigen aeroben Zersetzungsbedingungen geformt worden sein. Der Durit dagegen gelangte unter relative nässerer Kondition $\mathrm{zu}$ Ablagerung und ist anaeroben Zersetzungen unterworfen worden: weiterhin steht der Vitrit dazwischen.* Da die Kohle im allgemeinen aus diesen Kohlenstreifen besteht, variieren die oben beschriebenen Beziehungen in dem begrenzten Bereich (für eine länger andauernde Ablagerung pflanzlicher Stoffe). Es ist deshalb möglich, dass diese Urstreifenarten während der Zersetzungsvorgänge die abwechselnden Zersetzungsbedingungen durchlauften, entsprechend der $\mathrm{Ab}$ senkung und dem Aufstieg des Bodens. Infolgedessen entstehen die sehr verschiedenen Charakter zeigenden Streifenarten.** Nämlich wie aus der schematischen Darstellung beispielsweise zu ersehen, gelangt der "Urfusit" anfangs an trockenster Stelle zur Ablagerung. Kommt er aber ohne weiteres durch stärkere Absenkung in Räume des Luftsauerstoffabschlusses, so wird er nicht zum Fusit sondern zum Semifusit. Ein "Urvitrit" kann nach seiner Ablagerung sowohl die echte VitritZersetzungsbahn durchlaufen als auch eine Bahn, welche zum Durit oder Fusit hinführt. Infolgedessen entsteht am Ende der 1. Stufe der Inkohlung eine dem Vitrit ähnliche Streifenart, die aber hinsichtlich des optischen und physikochemischen Charakters dem Durit oder Fusit ähnelt. Diese Vorstellung darf auch für Durit gelten; obgleich der Durit die eigentlichen Mazeralen (d.h. Grundmasse, Exinit, Inerites) hat, zeigt er oftmals die optischen und physikochemischen Eigenschaften der Kannelkohle oder Boghead-Kohle.

Wurden die pflanzlichen Stoffe auf diese Weise in der ersten Stufe der Inkohlung in verschiedenen Graden zersetzt und dann weiter umgewandelt, so dürften die vielen Variationen in der gleichen "Urstreifenart" entstehen. Solche Unterschiede werden sich entsprechend dem Fortschritt der Inkohlung allmählich verstärken. Da die charakteristischen Unterschiede zwischen den Streifenarten zum Anthrazit allmählich nachlassen,*** könnte es auch möglich sein, dass die oben erwähnten Unterschiede der gleichen Streifenart nach der Erreichung eines bestimmten

* M. Th. Mackowsky behauptet, dass Vitrit unter den nässesten Bildungsbedingungen entstanden sei. Unter zunehmend trockneren Bedingungen folgt Durit und schliesslich Fusit.

** Absenkung und Aufstieg des Bodens beeinflusst nicht nur die biologische Zersetzung sondern auch die Azidität des Wassers und der verschiedenen Katalysatoren. Deren Veränderungen beschleunigen odor hemmen auch die Zersetzungswirkung-Reduktion, Oxydation und Hydrolyse.

*** Nach M. Th. Mackowsky erreicht die C- Anreicherung des Vitrits im Laufe der 1-Phase etwa $80 \%$, und des Fusits ca. 93\%. Demnach unterscheiden sie sich voneinander um fast $15 \%$ Kohlenstoff. Durch die 2-Phase der Inkohlung erfolgt eine Angleichung des C-Gehaltes vom Vitrit und Fusit. Der Unterschied beträgt höchstens noch etwa 1-2\%. Optisch wird auch diese Ausgleichung bestätigt. 
Inkohlungsgrades immer mehr verwischen und schliesslich verschwinden. Nach $\mathrm{H}$. STACH ist die Umwandlung der Kohle im Braunkohlestadium in erster Linie ein Kolloid-physikalischer Vorgang und während dessen hat die chemische Veränderung eine relativ geringe Rolle gespielt, weil der Kohlenstoff sich nur wenig anreichert. Mit H. Stach übereinstimmend kam M. TeICHMüLleR zum Schluss, dass die strukturelle Metamorphose der stofflichen vorausgeeilt ist. Andererseits ist die Umwandlung der Kohle im Steinkohlenstadium eine stoffliche Metamorphose, die durch eine zunehmende Aromatisierung der Huminkomplexe gekennzeichnet ist. Nach der Vorstellung von VAN KREVELEN besteht die Kohle aus aromatischen Ringgruppen und nichtaromatischen Brücken, welche die Ringgruppen miteinander verbinden. Im Laufe der Inkohlung werden die Brücken allmählich abgebaut, während die lamellenförmigen Aromatkomplexe immer breiter und schichtig, wabenartig werden.

Auch nach der Ansicht von HUCK und KARWELL hat die nichtaromatische Brücke ein wasserstoff- und sauerstoffreiches Methylensysthem, und aüssert sich die Metamorphose der Kohle in Sprüngen von Ketten dieser Methylensysthems. Abgesehen davon, ob dieses Methylensysthem auf Zellulose- oder Ligninabkömmlinge zurückgeht, wird übereinstimmend angenommen, dass im Laufe der Metomorphose die Seitenketten (nichtaromatisch) allmählich aufgebaut werden, und dass gleichzeitig die Vernetzung-der Kondendationsprozess-stattfindet und Brechindex, Reflexionsvermögen u.s.w. allmählich ansteigen. Bei diesem Fall ist es noch nicht klar, an welcher Stelle des aromatischen Kerns und mit welcher Länge die polaren Gruppen ansetzen.

Wie aus schematischen Darstellungen, die VAN KREVELEN für die Kohlengenese gezeigt hat, zu ersehen, hat Lignin viel H, O, R, u.s.w. als Seitenketten. Wenn es mehr oder weniger stärker oxydiert wird, dann werden die Seitenketten dementsprechend stärker abgebaut und damit unter der nachfolgenden Metamorphose schneller aromatisiert. Im Gegenfall würde die Aromatisierung unter gleichen Metamorphosefaktoren langsamer verlaufen.

Bei Berücksichtigung der chemischen Prozesse wird es also klarer, dass die oben erwähnten Aerobieverhältnisse für die Inkohlung von Bedeutung sind, besonders wegen ihres Einflusses auf die nachfolgende Metamorphose.

\section{b: Inkohlungsverlauf im Chikuho-Kohlenbecken-Chikuho-Inkohlungs-Typ}

Nun, nach den Ergebnissen der Kurzanalyse, der mikroskopischen und geologischen Untersuchung wird hier der Inkohlungsverlauf im Chikuho-Kohlenbecken zusammenfassend dargestellt.

Da die Kohle der Zeche Takamatsu in den untersuchten Kohlenflözen niedrigst inkohlt ist, kann man den Inkohlungsverlauf der Chikuho-Kohlen verfolgen, indem man damit den Inkohlungsgrad der beiden anderen Kohlenflöze vergleicht. Wie schon erwähnt, tritt während der Ablagerung der Tertiärformation in diesem 
Kohlenbecken die Schwankung der Absenkungsgeschwindigkeit des Bodens auf. Namentlich durch den NNW-SSE-Bruch, der die Tertiär- und Prätertiär-Formationen begrenzt, konnte der Ostteil im Vergleich zu dem Westen stärker absinken. Da der Durit gar keine auffallenden Unterschiede im Ausgangsstoff am Ost- und Westteil des Kohlenbeckens gezeigt hat, dürfte sich der Torf anfangs gleichartig im ganzen Becken ablagert haben. Demnach entsprechend der Schwankung der Bodenabsenkungsgeschwindigkeit dürfte der Torf des Ostteils relativ schneller dem nachfolgenden Inkohlungsprozess unterworfen worden sein. Diese Vermutung darf auch mit der Isopachenkarte bestätigt werden, welche die Mächtigkeitsveränderung zwischen dem Yoheda-Goshaku-Kohlenflöz und der obersten Grenze des YamagaSandsteins zeigt. Sinkt der Torf stärker, desto unregelmässiger sind die Mikrostreifen. Da die Kohle des Ostteils wegen der stärkeren Absenkung unvollkommen zersetzt worden ist, sind während der Inkohlung weniger nicht aromatische polare Gruppen vom Kern abgesprungen. Wenn-entsprechend dem Fortschritt der Inkohlung-diese nicht aromatischen Verbindungen zwischen Seitenkette und Kern oder Seitenketten untereinander durch die unvollkommen Zersetzung der 1. Stufe nicht so stark geschwächt worden sind, so konnte sich die Aromatisierung bei der nąchfolgenden Umwandlung verspäten.

Da die Entwässerung unter den Faktoren vorkommt, die im Vergleich zu der Devolatilisation von niedriger Ordnung ist, dürfte der Wassergehalt der betreffenden Kohlen schon normalen Ablauf der Inkohlung zeigen.

Auf diese Weise ist der Bruch, der an der Ostgrenze zwischen Tertiär und Prätertiär liegt, für die Inkohlung im Chikuho-Kohlenbecken von Bedeutung. Da die Kohlenflöze ausserdem noch von einigen anderen Brüchen durchsetzt werden, könnten Zweifel entstehen, ob diese Brüche auch Einfluss auf den oben beschriebenen Inkohlungsablauf haben. Da die Kohlenproben aber an durch diese Brüche ungestörten Stellen entnommen worden sind, kann man sagen, dass dieser Inkohlungsverlauf hauptsächlich auf den Grenz-Bruch zuruckzuführen ist. Wie schon erwähnt, sind die geologischen Bedingungen im Onoura- und Tagawa-Gebiet die gleichen wie in Takamatsu, und auch aus der Isopachenkarte des Onoura-Gebietes ist das gleiche Absenkungsverhältnis zu ersehen. Daher ist anzunehmen, dass die gleichartigen Inkohlungsphänome wie in Takamatsu-Gebiet auch in den beiden anderen Gebieten zu ersehen sind.

Im Diagramm aber ist die annormale Inkohlung in den Onoura- und TagawaKohlenproben nicht zu erkennen. Da die Kohlen dieser Gebiete älter und reifer sind, darf der Inkohlungsverlauf, wie Abb. 4 zeigt, von 1 zu 2 nach 3 u.s.w. allmählich fortschreiten. Zwischen der Otsuji- und Noogata-Stufe liegt eine Diskordanz, die auf eine orogenische Bewegung hinweist. Diese könnte die Ursache dafür sein, dass die Kohlenflöze der Noogata-Stufe, die früher einmal das der TakamatsuKohlen ähnliche Inkohlungsverhältnis besessen haben, heute die normalen Inkohlungserscheinungen zeigen. 
H. Matsushita hat die durch den grossen Bruch gekennzeichnete Struktur den Chikuho-Typ benannt. Da die gleiche Struktur noch in einigen Kohlenbecken in Kyushu auftritt, könnet man diesen Inkohlungsverlauf auch in anderen Kohlenbecken finden. Dieser Verlauf der Inkohlung ist mit der geologischen Struktur vom Chikuho-Typ innig verbunden und deshalb als "Chikuho-Inkohlungs-Typ" zu benennen.

\section{Zusammenfassung}

Bei der Untersuchung der Kohlen im Chikuho-Kohlenbecken wird es klar, dass die geologische Struktur und die Inkohlung miteinander sehr innig verbunden sind.

Die geologischen Vorgänge, welche die Struktur geschaffen haben, die H. MatsuSHITA als Chikuho-Struktur-Typ bezeichnet, könnten die Kohlen in der ersten Phase der Inkohlung am bedeutendsten beeinflusst haben.

Da die gleiche Struktur noch in einigen anderen Kohlenbecken in Kyushu auftritt, könnte der gleiche Inkohlungsverlauf-als Chikuho-Inkohlungstyp bezeichnet -auch in diesen zu erwarten sein.

\section{Schriftum}

NAGAO, T.: Palaeogene Coal bearing Formation of the Island of Kyushu, Sei. Rep. Tohoku Imp. Univ., Vol. 12, No. 1 (1927).

Matsushita, H.: Geology of the Coal Fields in Northern Kyushu, Sci. Rep. Kyushu Univ., Ser. D. Vol. 3, No. 1 (1949).

Univ., Ser. D, Vol. 3, No. 2 (1951).

StACH, E.: Der Inkohlungssprung im Ruhrkarbon, Brennstoff-chem., Bd. 34, H. 23/24 (1953).

Mackowsky, M. Th.: Inkohlung und Chemie der Kohle, Glückauf, 87, H. 23/24 (1951).

PATTEisky, K.: Veränderungen der chemischen und physikalischen Beschaffenheit von Vitriten und Mattkohlen beim Ablauf der Inkohlung, Brennstoff-Chemie, 34, 102/108 (1953).

: Die Veränderung der Steinkohle beim Ablauf der Inkohlung, Brennstoff-Chemie, 34, $5 / 6 \& 7 / 8$ (1953).

LehmanN, K. \& HofFmanN, Edw.: Neue Erkenntnisse über Bildung und Umwandlung der Kohle, Glückauf, 1932.

TeichmüLleR, M. \& R.: Inkohlungsfragen im Ruhrkarbon, Z. Geol. Ges., 99, 40-77 (1949).

: Die stoffliche und strukturelle Metamorphose der Kohle, Geol. Rdsch, 42, H. 2 (1954).

TEICHMÜLLER, M.: Zum petrographischen Aufbau und Werdegang der Weichbraunkohle, Geol. Jb., 64, 265-296 (1950).

TAKahashi, R.: Charaktereigenschaften der Kyushu-Kohle (Japan), Jour. Geol. Soc. Japan, Vol. 61, 723 (1955).

van Krevelen, W.: Physikalische Eigenschaften und chemische Struktur der Steinkohle, Brennstoff-Chemie, 34, 11/12 (1953).

\& SchuYer, J.: Coal Science, Elsevier Pub. Com., Amsterdam, (1957).

AsaI, K.: Zur Petrographie japanischer Kohlen, Proc. of the International Committee for Coal Petrology, No. 1, Aug. (1954).

SAKamoto, M.: Depositional Basin of the Palaeogene Sediments in Ebitsu-Miyata Area, Chikuho Coal Field, Jour. Min. Kyushu, Vol. 22, 12 (1954). 
R. TAKAHASHI

Die Beziehung zwischen der geologischen Struktur und der Inkohlung im Chikuho-Kohlenbecken, Japan.

\section{Tafeln}


Tafel 1 


\section{Erklärung der Tafel 1}

Öl : Öl-immersion.

MJ : Methylen-Jodid-Immersion (Einfgeführt von Prof. E. STACH). $\times 200, \times 480$ : originale Vergrössrung.

Abb. 1: gestörte Streifen, $\times 160$, Öl.

2: "I,$\times 380$, Öl.

3: $\quad$ " $\quad, \times 160$, Öl.

4: $\quad$ " $\quad, \times 160$, Öl.

5: $\quad \prime \prime \quad, \times 160$, Öl.

6: Fusinit, Semifusinit, fusinierte Vitrinit, $\times 160$, MJ.

7: $\quad$ " $\quad \times 160$, Öl.

8: $\quad$ " $\quad, \times 160$, MJ.

9: $\quad$ " $\quad, \times 160$, Öl. 

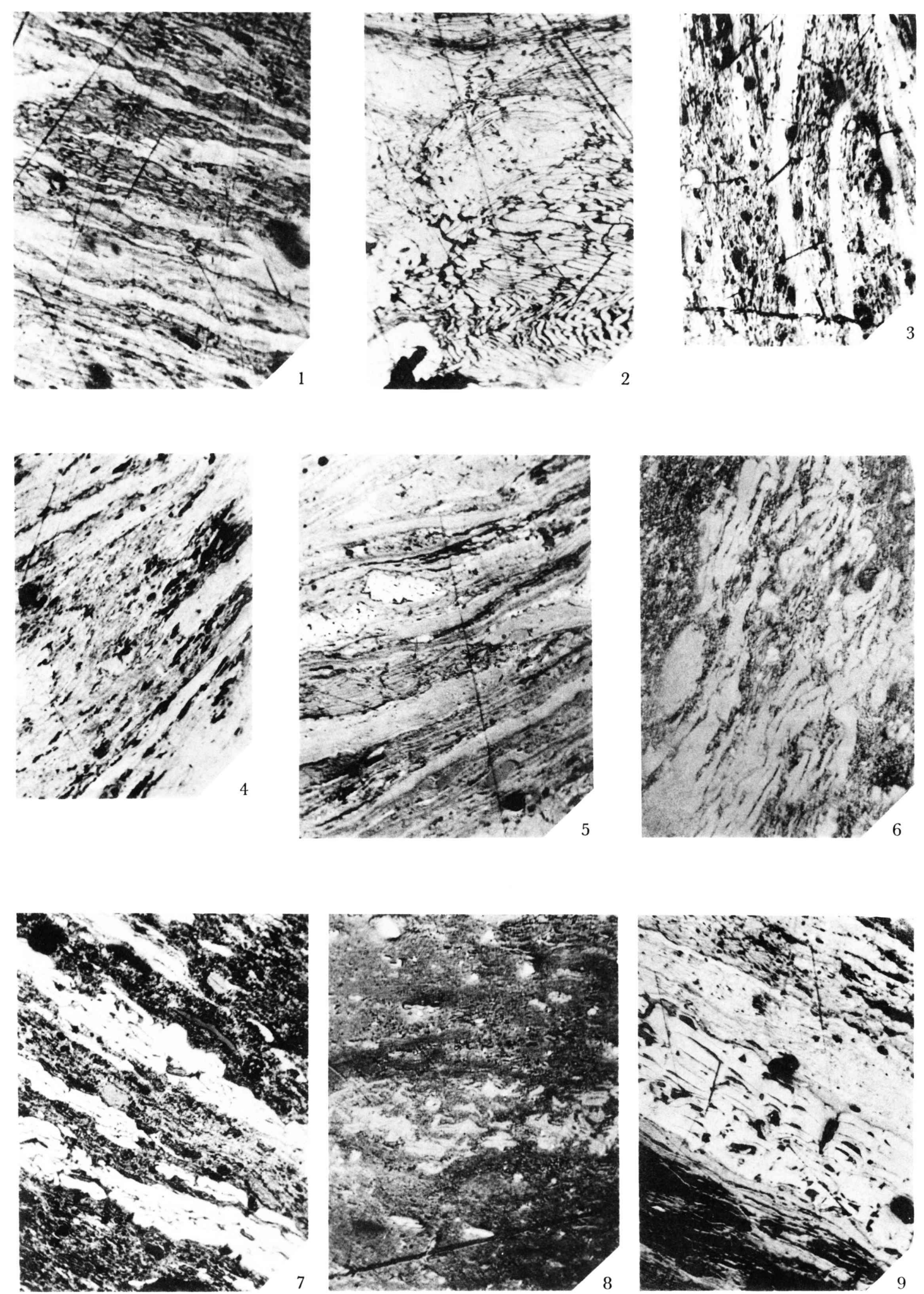

R. Takahashi: Die Beziehung zwischen der geologischen Struktur und der Inkohlung 
Tafel 2 
Erklärung der Tafel 2

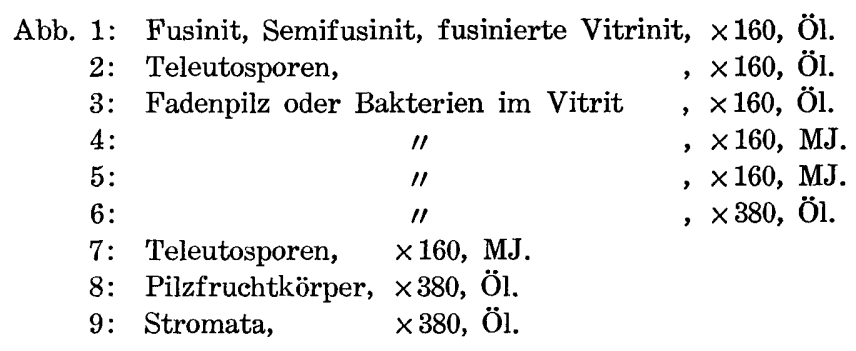



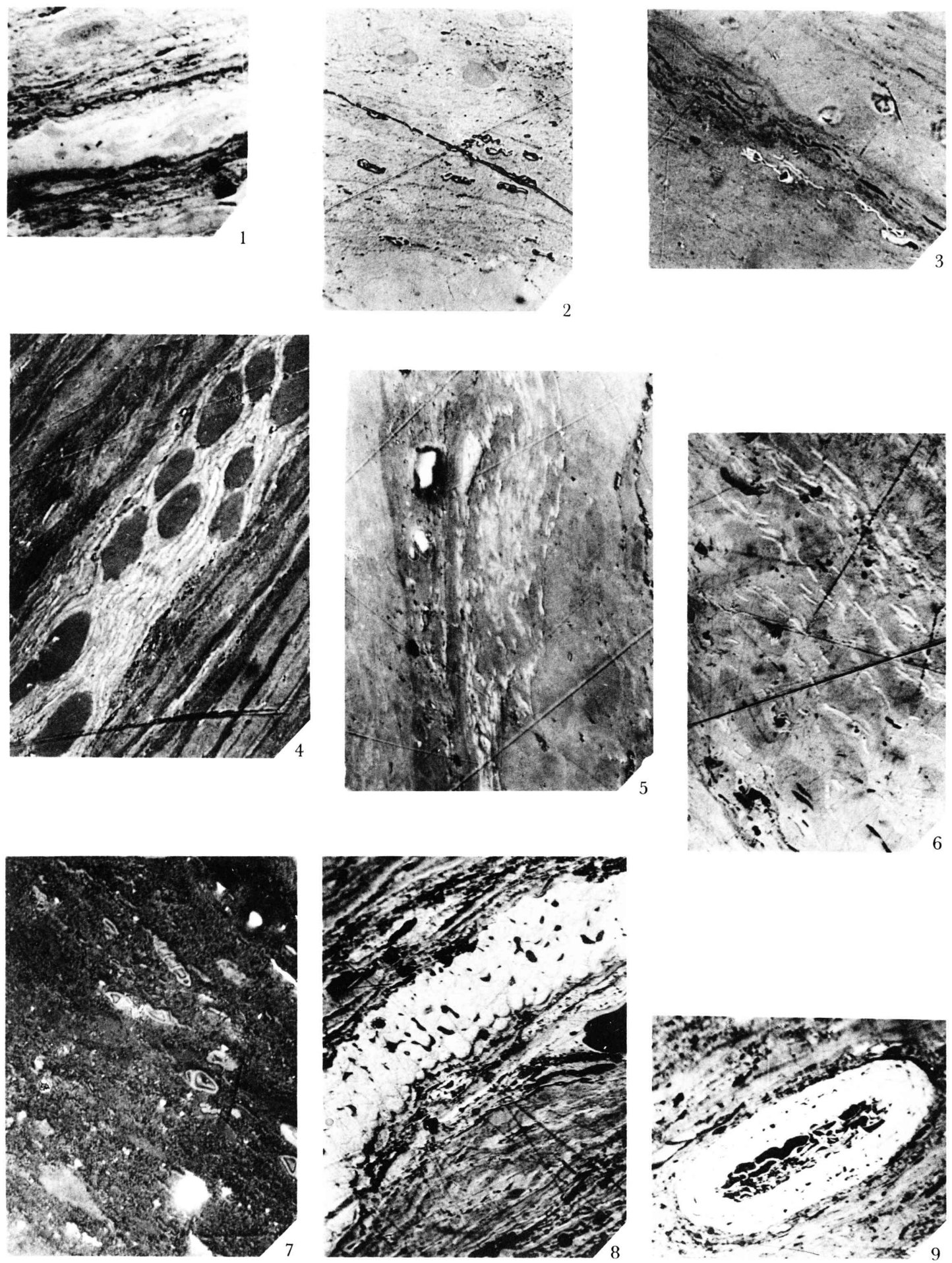

R. TAKahashi: Die Beziehung zwischen der geologischen Struktur und der Inkohlung 
Tafel 3 


\section{Erklärung der Tafel 3}

Abb. 1: Teleutosporen, $\times 380$, Öl.

2: $\quad$ " $\quad \times 380$, ÖI.

3: Pilzfruchtkörper, $\times 160$, Öl.

4: Sklerotium, $\times 380$, Öl.

5: grober Mikrinit, $\times 380$, Öl.

6: $\quad \prime \prime \quad, \times 160$, Öl.

7: imprägnierte Tonig-Substanz in Vitrit-Gefüge, $\times 160$, Öl. 

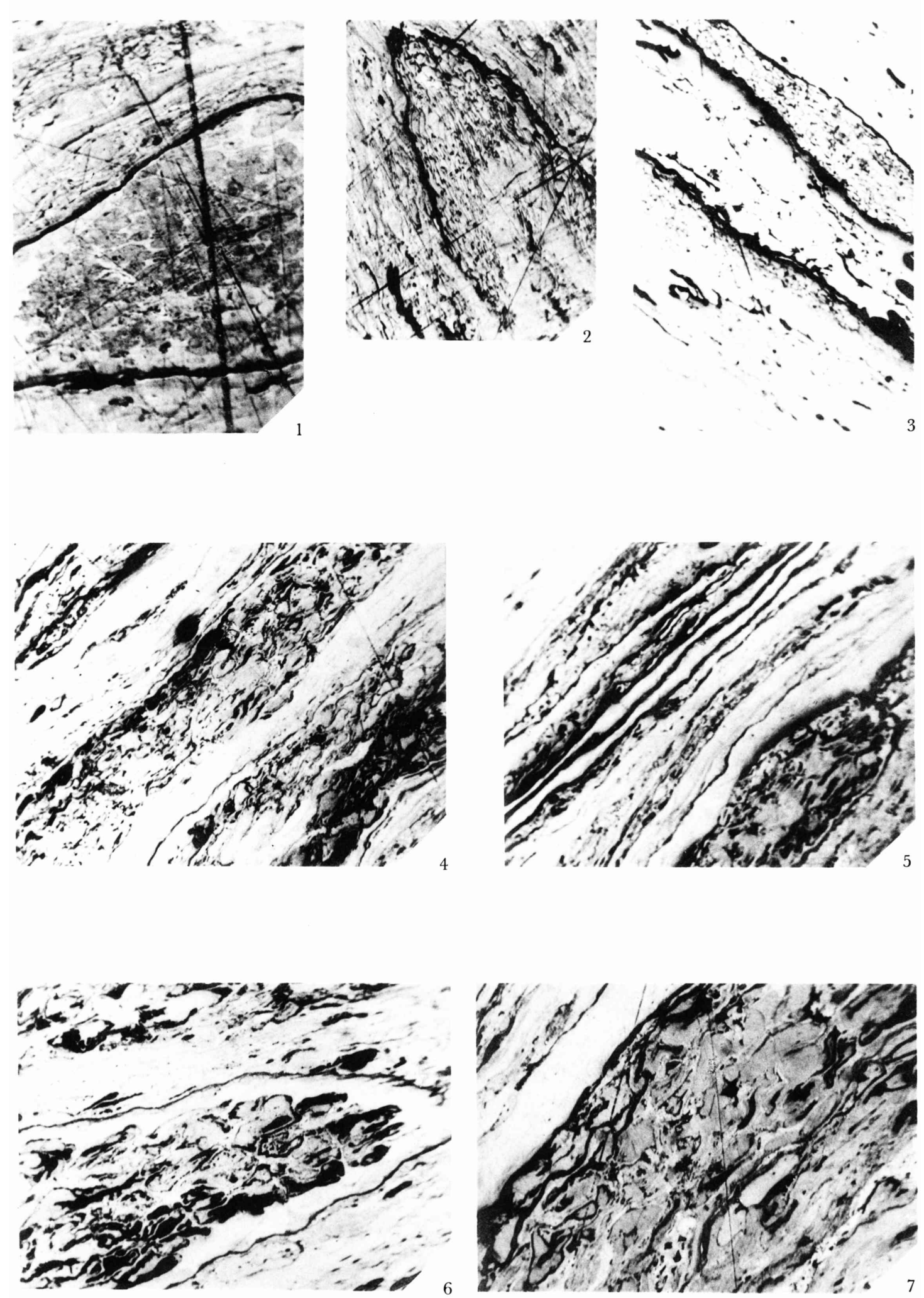

R. TAKahashi: Die Beziehung zwischen der geologischen Struktur und der Inkohlung 
Tafel 4 


\section{Erklärung der Tafel 4}

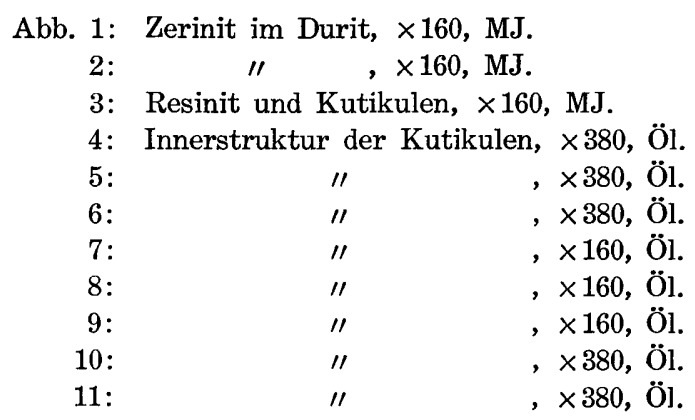



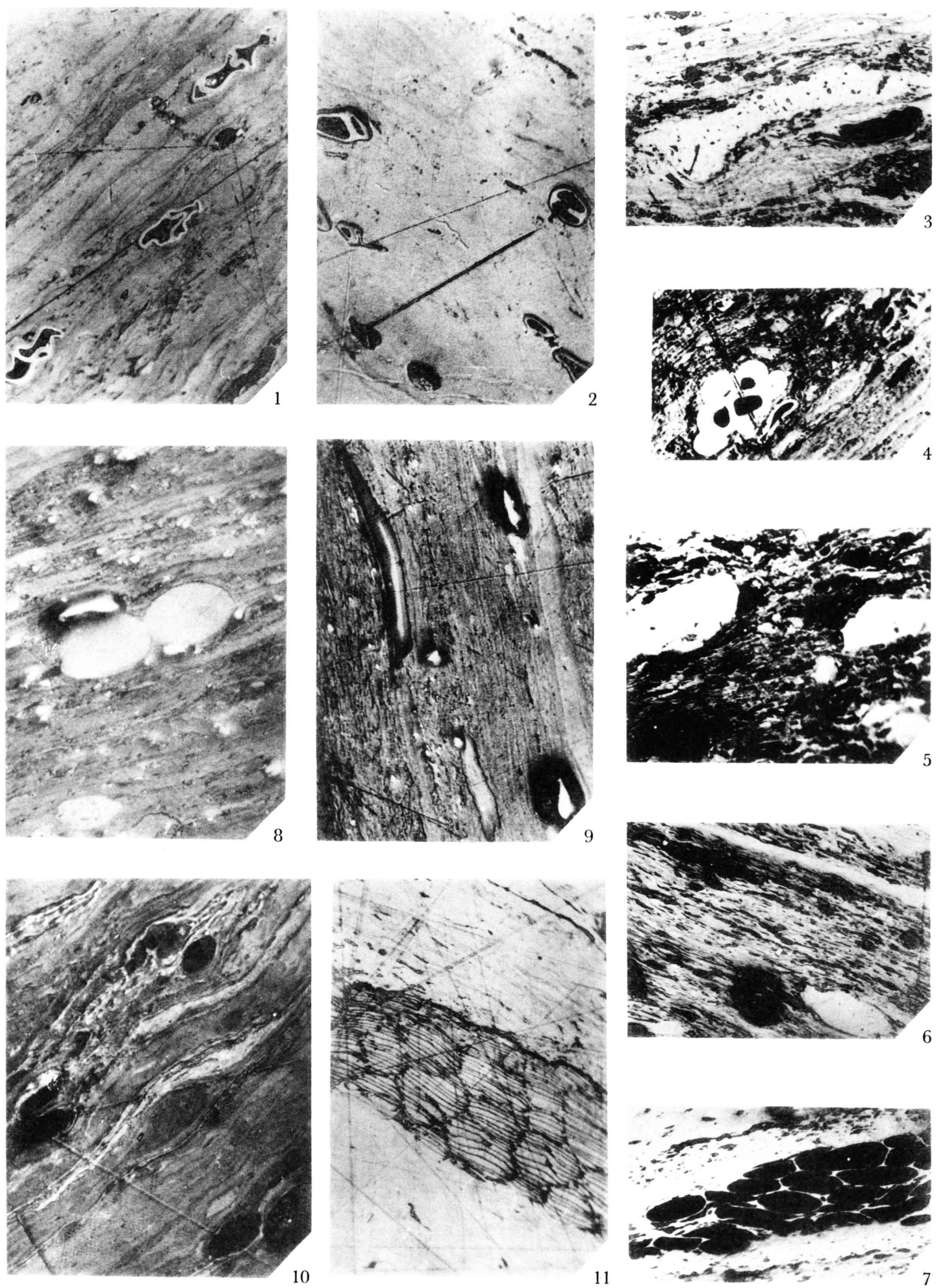

R. TAKahashi: Die Beziehung zwischen der geologischen Struktur und der Inkohlung 